\title{
Use of pitfall traps in the survey of edaphic fauna in the rural area of Traipu $-\mathrm{AL}$
}

\section{Utilização de armadilhas tipo pitfall no levantamento da fauna edáfica na zona rural de Traipu - AL}

Daniel de Souza SANTOS ${ }^{1}$, Carlos Henrique Vieira da SILVA*, Jadielson Inácio de SOUSA $^{3}$, Márcio Damasceno SANTOS ${ }^{4}$, Wesley de Oliveira GALDINO ${ }^{5}$

\author{
${ }^{1}$ Graduando em Ciências Biológicas na Universidade Estadual de Alagoas; E-mail: \\ carloshenriquevs@gmail.com; *In memorian Graduando em Ciências Biológicas na Universidade Estadual de \\ Alagoas; E-mail: daniel.biologo14@gmail.com; ${ }^{3}$ Graduando em Ciências Biológicas na Universidade Estadual \\ de Alagoas; E-mail: jadielsonsousa2017@gmail.com; ${ }^{4}$ Graduando em Ciências Biológicas na Universidade \\ Estadual de Alagoas; E-mail: marcio10010@hotmail.com; ${ }^{5}$ Graduando em Ciências Biológicas na Universidade \\ Estadual de Alagoas; E-mail: wesleygaldinobmx@gmail.com. \\ E-mail do autor correspondente: daniel.biologo14@gmail.com.
}

Resumo- Armadilhas do tipo pitfall são utilizadas no meio científico por ser de extrema importância, pois facilita na coleta de pequenos insetos que circulam na superfície terrestre, os chamados animais edáficos, que normalmente não são tão fáceis de capturar. Com este trabalho, objetivou-se identificar insetos típicos da fauna edáfica na zona rural de Traipu-AL. Para a realização do mesmo, foram dispostas quatro armadilhas do tipo pitfall, em um quadrante medindo $4 \mathrm{~m}^{2}$, com distância equivalente a $1 \mathrm{~m}$ de uma armadilha para a outra. $\mathrm{O}$ experimento foi realizado de abril a maio de 2020. O material coletado foi colocado em um recipiente com álcool 70\% e levado ao laboratório da Universidade Estadual de AlagoasUNEAL para que pudesse ser identificado. Portanto, foram coletados 316 insetos, sendo destes, 121 na armadilha "A", 50 na armadilha "B", 55 na armadilha "C" e 90 na armadilha "D”. Em geral, observou-se a predominância de formigas, cujas, pertencem à família Formicidae, da ordem Hymenoptera. Levando-se em conta o tempo que as armadilhas pitfall foram observadas, é notório a sua aplicabilidade, tanto no contexto econômico, quanto para estudos científicos, sendo uma possibilidade por se tratar de uma armadilha prática e de fácil manuseio.

Palavras-chave: Análise Faunística. Insetos. Solo.

Abstract- Pitfall traps are used in scientific circles, as they are extremely important, as they facilitate the collection of small insects that circulate on the earth's surface, the so-called edaphic animals, which are usually not so easy to capture. This work aimed to identify typical insects of the edaphic fauna in the rural area of Traipu-AL. To perform the work, four pitfall traps were arranged, in a quadrant measuring $4 \mathrm{~m}^{2}$, with a distance equivalent to $1 \mathrm{~m}$ from one trap to the other. The experiment was carried out in April 2020. The collected material was placed in a container with $70 \%$ alcohol and taken to the Laboratory of the State University of Alagoas-UNEAL for identification. Therefore, 316 insects were collected, of which 121 in trap "A", 50 in trap "B", 55 in trap "C" and 90 in trap "D". In general, there was a predominance of ants, which belong to the family Formicidae, of the order Hymenoptera. 
Taking into account the time that pitfall traps were observed, its applicability is notorious, both in the economic context and for scientific studies, being a possibility because it is a practical and easy to handle trap.

Keywords: Fauna Analysis. Insects. Ground.

\section{Introdução}

O solo é o habitat de um conjunto de organismos, cujas interações biológicas e suas atividades podem influenciar as características físicas, químicas e biológicas do solo de diferentes maneiras (ALVES et al., 2020). Uma das melhores formas de estudar insetos é realizar excursões para observação de seus hábitos e para coletá-los (SUTIL et al., 2017). O manuseio e a coleta revelam informações, por meio da observação direta, que, muitas vezes, não estão registradas em livros ou artigos científicos (SOUZA et al.,2016).

As armadilhas de solo, tipo pitfall, por sua vez, são especialmente voltadas para insetos que caminham sobre o solo por incapacidade de voo ou por preferência de habitat (ARAÚJO et al., 2010). Assim, a quantificação de insetos pode além de demonstrar a condição de sustentabilidade dos sistemas, indicar a necessidade da adoção e implantação de táticas de manejo, visando manter as populações em equilíbrio, sobretudo aquelas que causem danos às culturas de importância agrícola. (GLAESER et al., 2014).

Neste contexto, em levantamentos de organismos edáficos, um método de amostragem amplamente utilizado são as armadilhas de queda, que capturam insetos, principalmente aqueles que habitam o solo, onde caem e, uma vez coletados, não conseguem mais sair (FREIRE et al., 2011). As armadilhas formadas por recipientes plásticos, enterrados no solo até a borda, capturam pequenos vertebrados e invertebrados que estejam andando na superfície (SANTOS et al., 2019), as mesmas podem, ainda, conter líquido conservante ou não, dependendo do interesse em obter artrópodes vivos ou já mortos.

Ao utilizar líquido, deve-se adicionar à água sabão líquido e formol a $3 \%$, conforme recomendado por Costa Silva et al. (2014). A coleta de insetos, quando realizada de forma correta e responsável, não causa danos ambientais e nem desequilíbrio das espécies, pois o volume coletado pode representar uma ínfima parcela de suas populações (AZEVEDO \& TOLOTTI, 2015).A grande riqueza de espécies é atribuída a diversos fatores como o tamanho reduzido, sistema muscular eficiente, sistema sensorial e neuro motor mais organizado que outros invertebrados; grande capacidade reprodutiva e metamorfose corporal (GULAN \& CRANSTON, 2012).

Conhecemos quase um milhão de espécies de insetos, mas cientistas acreditam que exista algo entre 2,5 e 10 milhões de espécies, ou seja, não conhecemos nem metade delas, talvez muito menos (DELECAVE, 2011). Os insetos pertencem ao grupo dos artrópodes, ocupam uma grande variedade de nichos nos ecossistemas (ARAÚJO, 2019). Os artrópodes constituem o maior grupo do reino animal sendo composto por mais $75 \%$ de todas as espécies conhecidas. Aproximadamente 1.100 .000 espécies de artrópodes foram registradas e provavelmente o mesmo número ainda deve ser identificado e classificado (HICKMAN, 2013). 


\section{Revista Ambientale}

Revista da Universidade Estadual de Alagoas/UNEAL

e-ISSN 2318-454X, Ano 12, Vol. 12 (3), julho/outubro (2020).

Portanto, o objetivo deste trabalho foi identificar insetos típicos da fauna edáfica coletados através de armadilha tipo Pitfall na zona rural de Traipu-AL.

\section{Material e Métodos}

O trabalho foi realizado no período de abril a maio de 2020 na comunidade Olho D'água do Lima, localizada na zona rural de Traipu-AL (Figura 1). O município tem uma área territorial de 685,776 km² sendo vizinho dos municípios de Gararu, Girau do Ponciano e Canhoba. Traipu está situado a $22 \mathrm{~km}$ a Sul-Oeste de Girau do Ponciano a maior cidade nos arredores e possui 8 metros de altitude, contendo as seguintes coordenadas geográficas: Latitude: $9^{\circ} 57^{\prime} 30^{\prime \prime}$ Sul, Longitude: $37^{\circ} 0^{\prime} 36^{\prime \prime}$ Oeste.

Figura 1 - Imagem de satélite do município de Taipu-AL.

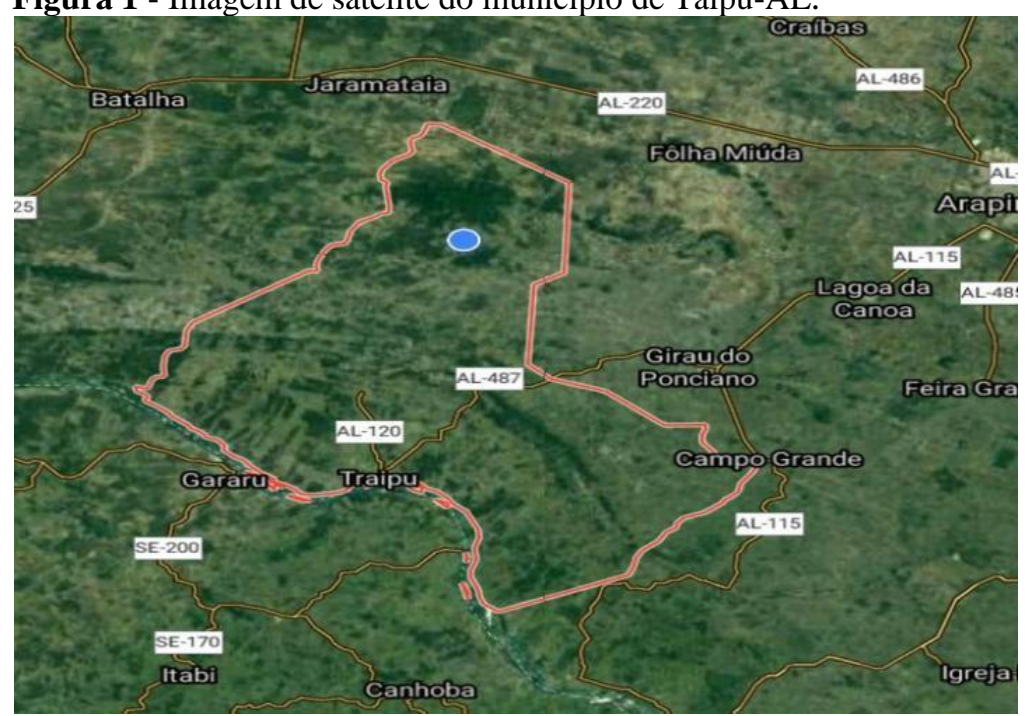

Fonte: Google Maps

O trabalho foi conduzido por uma semana onde foi destinada a coleta de insetos utilizando armadilhas pitfall. As mesmas foram colocadas, sem atrativos, em uma área de pastagem com vegetação abundante nos arredores (Figura 2). Em seguida, a equipe dedicouse a quantificar e identificar os insetos capturados, com o intuito de descobrir a ordem e a família de cada animal pertencente ao material etimológico coletado, fazendo-se uma análise comparativa, utilizando o livro Estudo dos Insetos de Triplehorn e Jonnson (2011) através de chaves ilustradas de identificação. 


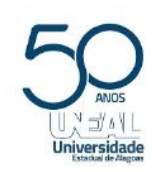

\section{Revista Ambientale}

Revista da Universidade Estadual de Alagoas/UNEAL

e-ISSN 2318-454X, Ano 12, Vol. 12 (3), julho/outubro (2020).

Figura 2 - Área de instalação das armadilhas pitfall.

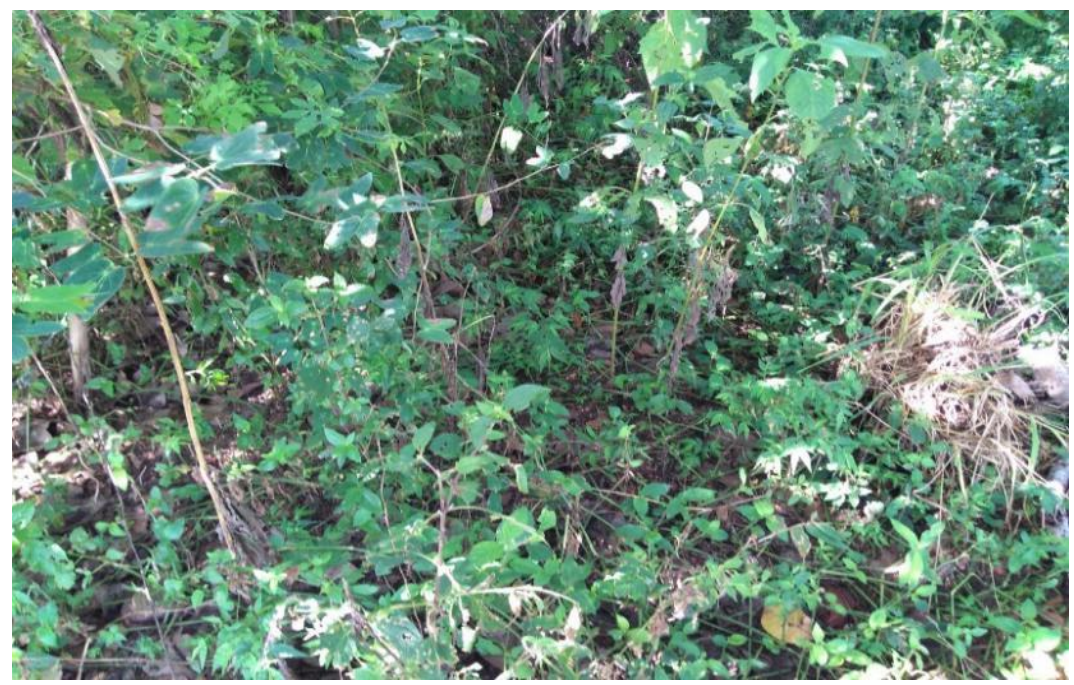

Fonte: Acervo dos autores.

Foram dispostas quatro armadilhas pitfall, em um quadrante medindo $4 \mathrm{~m}^{2}$, com distância equivalente a $1 \mathrm{~m}$ de uma armadilha para a outra. Na confecção das armadilhas utilizou-se quatro garrafas pets de 2 litros, cortadas na altura de aproximadamente $15 \mathrm{~cm}$ (figura 3), as mesmas foram enterradas com a borda ao nível do solo, em quatro buracos que foram cavados, para tal, foi necessário uma cavadeira, em seguida, adicionou-se em torno de $500 \mathrm{ml}$ de água e cinco gotas de detergente para quebrar a tensão superficial nas armadilhas. Para delimitar a área de coleta, foram utilizados gravetos introduzidos no solo e barbantes delimitando o perímetro, dividindo a área em quatro partes iguais (figura 4).

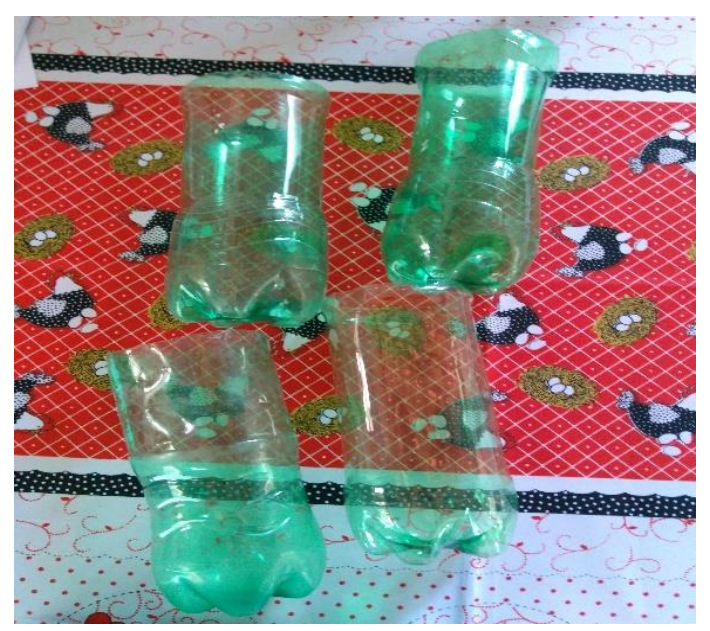

Figura 3 - Armadilha Pitfall.

Fonte: Acervo dos autores.

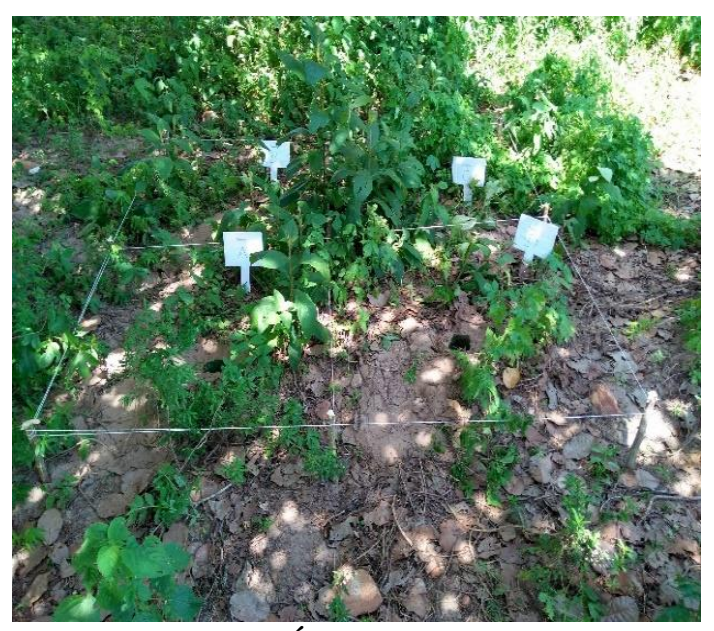

Figura 4 - Área de coleta demarcada. Fonte: Acervo dos autores. 
Revista da Universidade Estadual de Alagoas/UNEAL e-ISSN 2318-454X, Ano 12, Vol. 12 (3), julho/outubro (2020).

O material coletado foi posto em um recipiente (um recipiente para cada armadilha, totalizando quatro recipientes: ' $\mathrm{Ra}$; $\mathrm{Rb}$; Rc e Rd') em uma solução de álcool $70 \%$, onde os mesmos foram etiquetados. A análise e a identificação das espécies foram realizadas na Universidade Estadual de Alagoas-UNEAL, campus I, localizada no município de Arapiraca AL.

\section{Resultados e discussão}

Durante sete dias de levantamento, coletaram-se 316 insetos, sendo destes, 121 na armadilha "A", 50 na armadilha "B", 55 na armadilha "C" e 90 na armadilha "D" como mostra a tabela 1 .

Tabela 1- Coleta diária de insetos.

\begin{tabular}{ccccc}
\hline Dias & A & B & C & D \\
\hline $1^{\circ}$ & 3 & 5 & 0 & 22 \\
$2^{\circ}$ & 13 & 12 & 16 & 19 \\
$3^{\circ}$ & 28 & 11 & 1 & 3 \\
$4^{\circ}$ & 5 & 7 & 2 & 9 \\
$5^{\circ}$ & 35 & 11 & 17 & 0 \\
$6^{\circ}$ & 23 & 2 & 13 & 10 \\
$7^{\circ}$ & 14 & 2 & 6 & 27 \\
\hline Total por & & & & \\
armadilha & 121 & 50 & 55 & 90 \\
\hline Total de & & & $\mathbf{3 1 6}$ & \\
insetos & & &
\end{tabular}

Cabral et al., (2018), no seu trabalho sobre o levantamento da Entomofauna na cultura da pimenta de cheiro (Capsicum chinense L.) utilizando armadilha de pet colorida obtiveram resultados semelhantes com 340 espécimes pertencentes a 6 ordens e 22 famílias da classe Insecta.

Os organismos foram identificados em 10 ordens (Hymenoptera, Coleóptera, Araneae, Julida, Diptera, Orthoptera, Scorpiones, Phamatodea, Mantodea e Opiliones) e 21 famílias: (Sarcophagidae, Thespidae, Phasmatidae, Julidae, Scorpionidae, Vaejovidae, Acrididae, Romaleidae, Tettigoniidae, Gryllidae, Gryllotalpidae, Formicidae, Araneidae, Lycosidae, Theraphpsidae, Phalongodidae, Carabidae, Curculionidae, Elateridae, Scarabaeidae e Coccinellidae).

As ordens que mais predominaram foram: Hymenoptera, Coleóptera, Orthoptera e Araneae, como mostra a figura 5. 
Revista da Universidade Estadual de Alagoas/UNEAL e-ISSN 2318-454X, Ano 12, Vol. 12 (3), julho/outubro (2020).

Figura 5 - As diferentes ordens identificadas.

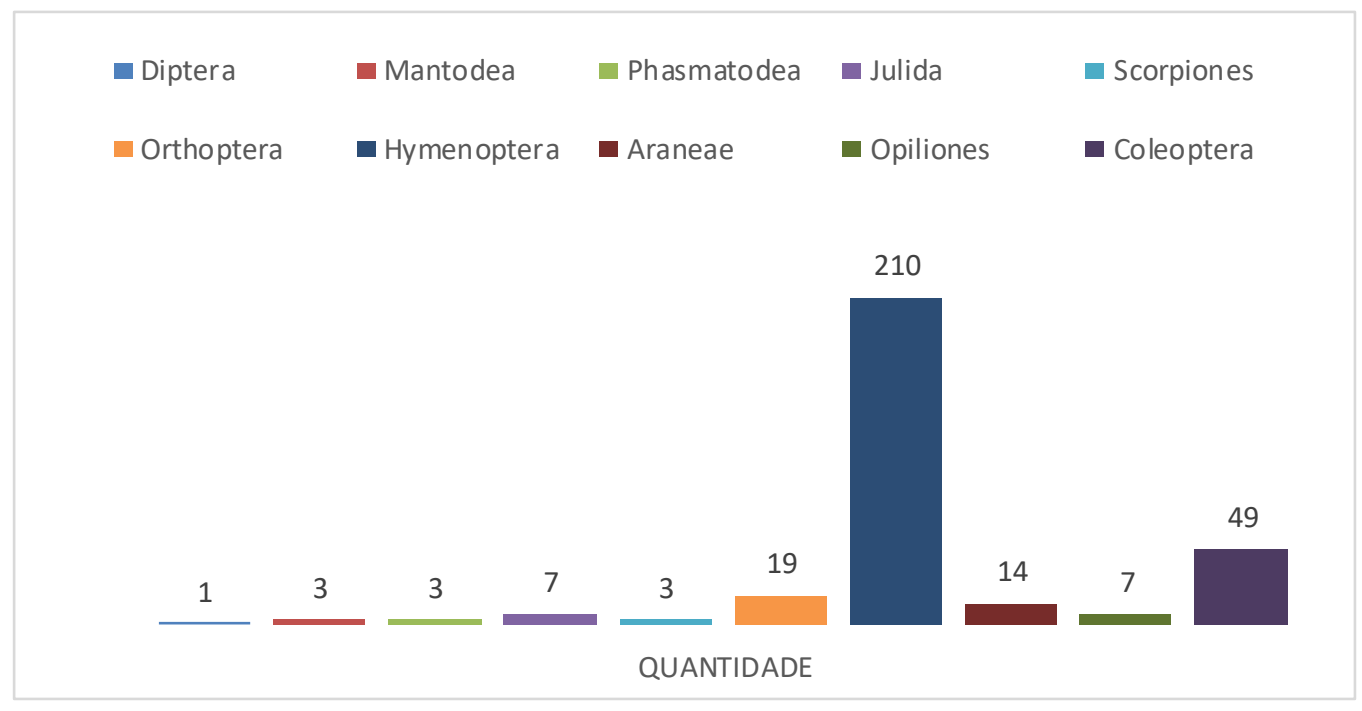

Em sua pesquisa, publicada na revista Globo Rural na edição de agosto de 2019 o pesquisador da Embrapa Maurício Antônio Lopes concluiu que: "Os insetos estão entre os organismos mais disseminados e abundantes no planeta, com cerca de 30 milhões de espécies, que juntas acumulam o maior volume de biomassa entre todos os seres vivos".

A importância dos insetos para a humanidade é incalculável, desde a polinização de plantas, a produção de mel e seda, o controle natural de pragas e o equilíbrio dos ecossistemas, até suas formas, cores e padrões, que tanto agradam aos nossos sentidos (LOPES, 2019).

Além da diversidade taxonômica, os insetos apresentam alta diversidade de comportamentos, como os diversos tipos de hábitos alimentares ou de locomoção; de tamanho e forma corporal (CORSO \& HERNÁNDEZ et al., 2011). Á medida em que ocorre o resgate da diversidade e equilíbrio ambiental, os insetos também respondem em diversidade e densidade, cumprindo a sua função indicadora (ARAÚJO,2019).

As famílias que mais predominaram foram: Formicidae, Scarabaeidae, Carabidae e Lycosidae, como mostra a figura 6. 
Figura 6: Diferentes famílias identificadas.

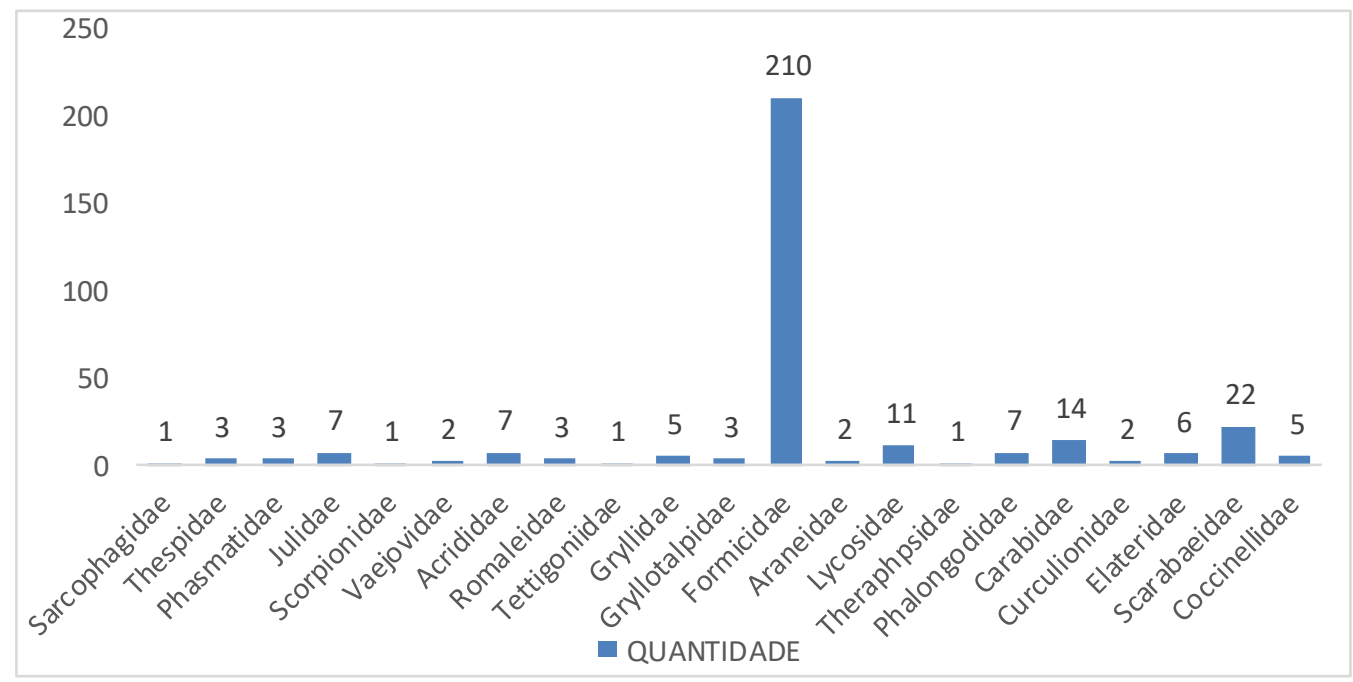

Em geral, no presente trabalho, observou-se a predominância de formigas, cujas, pertencem à família Formicidae, da ordem Hymenoptera, além desses animais contribuírem para o aumento da infiltração da água, eles mantêm o ambiente saudável, mantendo funções importantes para os ecossistemas naturais.

Segundo Souza et al. (2016), sua importância se deve principalmente ao comportamento eusocial aliado a complexos sistemas de comunicação, que permitem às formigas recrutarem companheiras e defender recursos com grande eficiência.

\section{Agradecimentos}

In memoriam de Carlos Henrique Vieira da Silva, grande amigo, parceiro e irmão, graças a seu empenho e dedicação conseguimos elaborar as armadilhas, sem ele não seria possível a realização deste presente trabalho. Deixamos aqui toda nossa honra e gratidão de termos conhecido e trabalhado com este ser humano incrível.

Ao Professor Dr. Rubens Pessoa de Barros, líder do grupo de estudos ambientais e etnobiológicos pela orientação desse trabalho.

\section{Conflito de interesses}

Os autores deste manuscrito não declararam conflitos de interesse. 


\section{Conclusão}

Levando-se em conta o tempo que as armadilhas foram observadas, foi possível identificar uma considerável diversidade de ordens e famílias, sendo então eficientes na captura de animais edáficos.

É notório a sua viabilidade, principalmente no contexto acadêmico pelo fácil manuseio, mas importante ressaltar que o local e o solo onde as armadilhas forem instaladas devem ser bem escolhidos.

\section{Referências}

ALVES, Carla et al. Caracterização da entomofauna em horta medicinal agroecológica utilizando armadilhas tipo pitfall. Caderno Verde de Agroecologia e Desenvolvimento Sustentável, v. 10, n. 1, p. 12, 2020.

ARAÚJO, R. C. S. et al. Entomofauna da Área de Proteção Ambiental Morros Garapenses: Conhecimento e Educação Ambiental. Revista Brasileira de Meio Ambiente, v. 7, n. 2, 2019.

AZEVEDO FILHO, W. S.; TOLOTTI, A. Os Insetos e a Ciência na Escola. Caxias do Sul: EDUCS, 2015.

ARAÚJO, C.C. et al. Comparação da abundância de invertebrados de solo por meio da estimação intervalar encontrados em diferentes ambientes na cidade de Ituiutaba - MG.

Bioscience Journal, v. 26, n. 5, p.817-823, 2010.

CABRAL, M. J. et al. Levantamento da Entomofauna na cultura da pimenta de cheiro

(Capsicum chinense L.) utilizando armadilha de pet colorida. Revista Ambientale, v. 10, n. 3, p. 52-60, 2018.

COSTA SILVA, V. et al. Escarabeídeos (Coleóptera: Scarabaeidae) de campo e floresta da Reserva Biológica de São Donato, Rio Grande do Sul, Brasil. Biotemas, v. 27, n. 4, p. 63- 71, 2014.

CORSO, G. \& HERNÁNDEZ, M. I. M. Borboletas frugívoras da Mata Atlântica no Parque Estadual da Serra do Tabuleiro, Santa Catarina, Brasil. Biotemas (UFSC), v. 25, p. 139-148, 2012.

DELACAVE, B. Insetos e a biodiversidade. 2011. Disponível em: <http://www.invivo.fiocruz.br/cgi/cgilua.exe/sys/start.htm?infoid=1133\&sid=2>. Acesso em: 6 de jun. 2020. 
Revista da Universidade Estadual de Alagoas/UNEAL e-ISSN 2318-454X, Ano 12, Vol. 12 (3), julho/outubro (2020).

FREIRE, E.S. et al. Eficácia de armadilhas pitfall no controle de Neocurtila sp. (Orthoptera: Grillotalpidae) em hortas orgânicas. V.6, n.2, p. 1-4, 2011. Trabalho apresentado no CONGRESSO BRASILEIRO DE AGROECOLOGIA, 7., 2011, Fortaleza. Resumos.

GULAN, P. J. \& CRANSTON, P. S. Os insetos: um resumo de entomologia. São Paulo: Roca, 2012. 440 p.

GLAESER, D. F.et al. Avaliação da Entomofauna em um sistema de consorciação de bananeira com plantas de cobertura, sob transição agroecológica, Cadernos de Agroecologia, v. 9 , n. 4, p. 1-12, 2014.

HICKMAN, C. P. Princípios Integrados de Zoologia. Rio de Janeiro, Guanabara Koogan, 2013, 423 p.

LOPES, M. A. Insetos na Dieta. Brasília, DF: EMBRAPA, 2019. Disponível em: < https://www.embrapa.br/busca-de-noticias/-/noticia/45712402/artigo---insetos-na-dieta > Acesso em: 08 de Jun. 2020.

SANTOS, R. S.; DE SOUZA, C. S.; RUFINO, C. P. B. Escarabeídeos (Coleóptera: Scarabaeidae) coletados em armadilhas pitfall em um remanescente florestal no município de Plácido de Castro, AC. Embrapa Acre-Artigo em periódico indexado (ALICE), 2019.

SOUZA, C. S. et al. Fauna de Formicidae capturadas com armadilhas Pitfall em remanescente florestal no município de Plácido de Castro. In: Embrapa Acre-Resumo em anais de congresso (ALICE). In: CONGRESSO REGIONAL DE PESQUISA DO ESTADO DO ACRE, 2.; SEMINÁRIO DE INICIAÇÃO CIENTÍFICA DA UFAC, 25., 2016, Rio Branco/Cruzeiro do Sul. Inovação: sustentabilidade e desenvolvimento regional: anais. Rio Branco, AC: Edufac, 2016.

SUTIL, W. P. et al. Besouros escarabeídeos (Coleóptera: Scarabaeidae) coletados com armadilhas pitfall iscadas em Rio Branco, AC. In: Embrapa Acre-Resumo em anais de congresso (ALICE). Caderno de Ciência e Tecnologia, Rio Branco, AC, v. 2, p. 67-68, 2017., 2017.

TRIPLEHORN, C.A; JONNSON, N.F. Estudo dos insetos. São Paulo: Cengage Learning. 2011, 809p. 